Evaluation of Parental-Type Classification for the Inbred-Backcross

Method of Estimating Gene Number

by

G.A. Rau,

C.E. McCulloch

and

M.A. Mutschler

BU-1095-MC

April 1993 


\title{
Evaluation of Parental-Type Classification for the Inbred- Backcross Method of Estimating Gene Number
}

\author{
G. A. Rau, C. E. McCulloch, and M. A. Mutschler \\ Department of Plant Breeding and Biometry, 252 Emerson Hall, Cornell University, \\ Ithaca, NY 14853 USA
}

Acknowledgment: This work was supported in part through Hatch Project NYC-149484. The manuscript benefited from a review by Mark Sorrells.

Running Title: Evaluation by inbred-backcross method 


\begin{abstract}
$\underline{\text { Abstract }}$
An attempt was made to estimate gene numbers for four quantitative fruit traits in tomato using the inbred-backcross method developed by Wehrhahn and Allard (1965). This led to a critical review of the method as used in publications to date. Estimation of gene number via the inbred-backcross method depends on the classification of lines into parental and non-parental types. Examination of prior publications shows that in several papers the classification of lines was done using tolerance intervals or ellipses rather than confidence regions. This would result in an underestimation of the gene number. No genes could be detected for any of the tomato fruit traits assayed except first color date using previous methods of line classification. For reasons discussed, we suggested use of confidence rectangles to classify lines as being parental and non-parental. Using this method, 14, 20, 25, and 30 genes were obtained, respectively, for fruit firmness, first color date, soluble solids, and fruit weight. For reasons discussed, these could be underestimations of actual numbers of genes affecting these traits. This study has implications concerning the precision of gene number estimates by other methods. Estimates of gene number similar to those previously published for the inbredbackcross method have been obtained using other methods, suggesting that the alternative methods may also be inaccurate.
\end{abstract}


The inbred-backcross method was developed by Wehrhahn and Allard (1965) as a means of estimating the number of genes involved in a quantitative trait. In this method a series of inbredbackcross lines is developed by crossing two parents to produce an $F_{1}$, which is backcrossed to the recurrent parent. A number of backcross individuals may again be backcrossed one or more times to the recurrent parent, then each line is advanced by single seed descent (SSD) until the desired degree of homozygosity is reached. $B C_{k} S_{j}$ will signify a line backcrossed $k$ times and selfed for $j$ generations. Wehrhahn and Allard (1965) plotted the mean of each line in $B C_{2} S_{4}$ against the mean of the same line in $B C_{2} S_{3}$. They identified non-parental lines based on a tolerance ellipse drawn around the mean of the recurrent parent. Lines whose means fell outside the ellipse were considered non-parental. Ellipses of the same size as the parental ellipse were used to delineate putative non-parental groups.

Several authors have reported success using the inbred-backcross method to estimate the number of major genes by which the two parents differed (Wehrhahn and Allard 1965, Talukdar 1972, Wu et al. 1975, Baker 1977, Thurling and Vijendra Das 1979, Sullivan and Bliss 1985b, Owens et al. 1985, Wehrhahn and Tai 1988, Chay and Thurling 1989). Other reports, often looking at other traits in the same experiments, could not distinguish any major genes (Wehrhahn 1964, Wu et al. 1975, Baker 1978, Russell and Stuber 1983, Sullivan and Bliss 1983a).

A series of inbred-backcross lines were developed to test the use of the inbred-backcross method to transfer a series of single genes (Rau 1989) and estimate gene number for complex fruit traits in tomato, including date to first ripe fruit, fruit weight, fruit firmness, and soluble solids. This project led to a critical examination of the inbred-backcross method and how it has been used to estimate gene numbers. There are a number of problems with the inbred-backcross method and the way it has been used (Baker 1978, Mulitze and Baker 1985a, Rau 1989). Mulitze and Baker (1985a) proposed a modification of the original method which resolves several of these, including lack of discontinuities in the data, overlap of the ellipses, lack of inclusion of data points in any ellipse, and determination of the number of genes responsible for a particular non-parental group. Two major flaws in the analysis methodology still exist involving the means of classifying offspring lines into parental and nonparental types. First, tolerance ellipses drawn about the mean of the recurrent parent have been used 
to achieve this classification. Second, each classification of offspring lines has been treated as a separate comparison, where an overall procedure may be better. All authors since Baker (1977) state they have used confidence ellipses or intervals, but comparison of their figures with the error variances given shows that each used a tolerance ellipse or interval, with the possible exception of Russell and Stuber (1983) who did not report their error variance (Rau 1989).

This paper illustrates the use of confidence regions for paired and unpaired data. The use of confidence regions is compared to the use of tolerance regions. Procedures were developed to control the overall probability of misclassifying lines as non-parental. Finally, the protocols were demonstrated using data generated from the study of four tomato fruit characteristics.

\section{Material and Methods}

\section{Plant Materials}

A population of inbred-backcross lines was developed following the procedure of Wehrhahn and Allard (1965). A donor plant carrying the slow-ripening mutant alcobaca (alc) was crossed with and backcrossed to Calypso. Fifty-six $B C_{1}$ plants were individually backcrossed to Calypso and advanced by three generations of single seed descent to produce a series of $55 B C_{2} S_{3}$ lines, since one line was lost in the process of selfing. These $B C_{2} S_{3}$ lines were planted in the field in 1985 as a randomized complete block with three blocks. Each of the offspring lines from each population was represented by one plot of four plants in each block. Each parent was represented by three plots per block. $F_{3}$ offspring of the original donor plant were used to represent the donor. Plant spacing was six feet between rows, three feet between plants, with six feet between every second plot to facilitate movement and finding plots in the field. The experiment was repeated in 1986 with $B C_{2} S_{4}$ progeny of a random plant from each line.

\section{Quantitative Traits Assayed}

Date of first fruit color was recorded for each plant as a measure of earliness, with August 1 arbitrarily designated as day 1 . Three ripe fruit were harvested from each plant and measured for weight, firmness, and soluble solids. Fruit were collected in the field and brought into a field building 
at least several hours before testing to minimize the effect of temperature on firmness and soluble solids measurements. All fruit were tested within 24 hours of harvest. Weight was measured on a Mettler top-loading balance to the nearest gram. Firmness was measured using a penetrometer modified for deformation testing with a $500 \mathrm{~g}$ weight (Bourne, 1973) in $0.1 \mathrm{~mm}$ units. A V-shaped base was used to minimize the effect of locule position on the measurements (Brady, CSIRO, Division of Rood Research, Australia, personal communication). Soluble solids were measured in degrees Brix using AO model 10430 temperature-compensated hand-held refractometers. The mean square error from the analysis of variance for a larger experiment of which this is a part (Rau and Mutschler, unpublished data) was used as the best estimate of environmental variance for each trait. Bartlett's test of homogeneity of variance (Snedecor and Cochran, 1980) and plots of standard deviation as a function of mean were performed on each quantitative trait for each year. Because the standard deviation increased linearly with the mean for weight, a $\log _{10}$ transformation was used to obtain homogeneous variance. Soluble solids showed heterogeneity of variance in 1985 , but not 1986 . There was no correlation between variance and mean and no transformation improved the homogeneity of variance in 1985. Firmness, especially in 1985 , and color date showed trends of increasing variance with mean, but were declared non-significant by Bartlett's test. For each trait the line mean in 1986 was graphed as a function of the mean of the same line in 1985 .

\section{Tolerance Ellipse}

The theory behind using the inbred-backcross method to determine the number of genes by which two lines differ is based on a binary classification of inbred-backcross lines into parental and non-parental types (Mulitze and Baker 1985a). This classification has been done using a tolerance ellipse. Inbred-backcross lines falling within the probability ellipse have been considered to have the parental genotype for that trait, while those outside have been considered to differ by one or more genes.

A tolerance ellipse about the mean of the recurrent parent was constructed by calculating the values of $x$ and $y$ that satisfy the formula 


$$
\frac{(x-\bar{x})^{2}}{s_{x}^{2}}+\frac{(y-\bar{y})^{2}}{s_{y}^{2}}=\left(1+\frac{1}{n}\right)\left(\frac{2 d f}{d f-1}\right)\left(F_{2, d f-1, .05}\right)
$$

where $\bar{x}$ and $\bar{y}$ are the mean of the recurrent parent in 1985 and 1986 respectively, $s_{x}^{2}$ and $s_{y}^{2}$ are the sample variances for each year, $n$ is the number of observations in the mean each year, and $d f$ is the degrees of freedom for estimating the variances (Johnson and Kotz 1972, p.68). This is analogous to the method used by Wehrhahn and Allard (1965) and others, except that they used $\chi_{2, .05}^{2}$ on the right-hand side of the equation. Our ellipse approximates theirs for large $n$ and large $d f$. Talukdar (1972) may have used some other value, based on the length of the axis of the probability ellipse, but how he calculated the ellipse is not completely described. $\chi^{2}$ would be appropriate if the true variance were known, and is a fairly good approximation for experiments with high degrees of freedom. The formula given here would be exact if we had pairs of data and included a cross product (covariance) term in $x$ and $y$. However, in our experimental design with single-seed descent, the data were independent within a line from year to year rather than paired, and therefore, the covariance term is zero. Although not exact, this approach is slightly more conservative than $\chi^{2}$ and provides a better approximation since the true variance is unknown. The conservativeness in [1] arises because $2 F_{d f-1, .05}^{2}>\chi_{2, .05}^{2}$ and the other multipliers are greater than 1 . Mean values for $n$ and $d f$ for the two years were used to give a conservative estimate. In this experiment, the value of the right-hand side

was 6.04 for all traits except color date, for which it was 6.17 ; using $\chi^{2}$ the value of the right-hand side would be 5.99 .

Statistically the $95 \%$ tolerance ellipse represents the range within which we would expect the sample values to fall $95 \%$ of the time if we were to draw repeated samples from the same population. Thus, it represents the range of plausible values for the population. That is not the same as asserting that values within the ellipse are not significantly different from the mean of the recurrent parent, the center of the ellipse, nor is it a test of equality with the recurrent parent.

\section{Confidence Ellipse}

A confidence ellipse delineates the range around a sample mean within which the true mean is expected to lie, with a certain level of probability. A 95\% confidence ellipse represents the range in 
two dimensions, within which, the true mean falls with a $5 \%$ chance of error. It can be used to test the hypothesis that two population means are identical.

Confidence ellipses were calculated about the mean of the recurrent parent. The formula is identical to the one given above for probability ellipses, except that the right hand side is multiplied by $1 / n$, where $n$ is the number of observations used to calculate the mean. Where $n$ differed in the two years due to dead or off-type plants the mean $n$ was used.

\section{Two-Sample $t$-Test}

Hotelling's $T^{2}$, a multivariate, pooled, two-sample $t$-test (Johnson and Wichern, 1988), was used to test the hypothesis that the means of lines in the inbred-backcross population were statistically different from the recurrent parent for each trait. It is calculated as

$$
\frac{\left(\bar{x}_{p}-\bar{x}_{\ell}\right)^{2}}{s_{x}^{2}\left(\frac{1}{n_{x p}}+\frac{1}{n_{x \ell}}\right)}+\frac{\left(\bar{y}_{p}-\bar{y}_{\ell}\right)^{2}}{s_{y}^{2}\left(\frac{1}{n_{y p}}+\frac{1}{n_{y \ell}}\right)}=T^{2}
$$

where $\bar{x}_{p}$ and $\bar{x}_{\ell}$ are the means of the parent and offspring lines, respectively, in 1985 with other notation similar to that above. Again, we have set the cross product term equal to zero since, in our design, the covariance is zero. The null hypothesis that the means are equal was rejected at the $95 \%$ confidence level for

$$
T^{2}>\left(\frac{2 d f}{d f-1}\right)\left(F_{2, d f-1, .05}\right)
$$

where $d f$ is the mean degrees of freedom used to estimate the variance.

\section{Rectangular Tests}

All of these methods are approximate in our experiment because they are based on the assumption of paired data (each datum in one year or, more generally, one variable is paired with one datum in the second year). With single seed descent it is impossible to have paired data since for each line all the plants in the second year are derived from one plant in the preceding generation. We now derive the rectangular tests which are exact tests of significance or equality in the current experiment.

A rectangular area corresponding to the tolerance ellipse was constructed by drawing a 
probability interval $\left(\bar{x}_{p} \pm t s_{x}\right)$ about the mean for each year independently. To give an overall $\alpha$ rate of 0.05 the $\alpha_{y}$ rate used for each year was 0.0253 , calculated as

$$
1-\alpha=\left(1-\alpha_{y}\right)^{2} .
$$

This area was called a 'tolerance rectangle'. A similar 'confidence rectangle' was drawn, using $\bar{x}_{p} \pm t s_{x} / n^{\frac{1}{2}}$ to delineate the rectangle.

A test which we call a 'two-sample $t$ rectangle test' was performed by conducting two-sample tests of significance for each year independently. The $\alpha_{y}$ rate used for each year was again 0.0253 to give an overall rate of 0.05 . The hypothesis that the recurrent parent and inbred-backcross line were the same in a given year was rejected if

$$
\frac{\left|\bar{x}_{p}-\bar{x}_{\ell}\right|}{\left(s_{x}^{2}\left(\frac{1}{n_{p}}+\frac{1}{n_{\ell}}\right)\right)^{\frac{1}{2}}}>t
$$

for that year (Snedecor and Cochran 1980). They were declared significantly different overall if the hypothesis was rejected either year. The rectangular method could be applied to any other one variable statistical procedure, for example two-sample rank tests, one-tailed tests, or multiple range tests.

Another flaw of the methods used to classify lines as parental or non-parental is that the error rate, $\alpha$, only applies to a single comparison of an offspring line to a parental line. In many situations it would be desirable to control the overall rate of misclassification since conclusions about gene number depend on all the classifications. This can be done by replacing the $t$ table value by an appropriate percentage point from a multivariate t-distribution. Details are given in the Appendix.

\section{Estimation of Gene Number}

Mulitze and Baker (1985a) showed that the number of loci at which the donor and recurrent parent differ for each trait can be estimated by

$$
\hat{g}=\ln (1-\hat{d}) / \ln (1-P)
$$

where $\hat{\boldsymbol{g}}$ is the estimated number of loci and $\hat{d}$ is the proportion of lines that differ from the recurrent parent. With two backcrosses, the probability, $P$, that any inbred-backcross line will differ from the 
recurrent parent is 0.125 . Mulitze and Baker (1985a) used the formula

$$
\hat{d} \pm 1.96[\hat{d}(1-\hat{d}) / \ell]^{\frac{1}{2}}
$$

where $\ell$ is the number of inbred-backcross lines, as a $95 \%$ confidence interval for $\hat{d}$.

A better approximation than [6] for a $95 \%$ confidence interval for $d$, is

$$
\hat{d}=\frac{c \pm .5+z^{2} / 2 \pm z \sqrt{(c \pm .5)-(c \pm .5)^{2} / \ell+z^{2} / 4}}{\ell+z^{2}}
$$

where $c$ is the number of lines that differ from the recurrent parent, $\ell$ is the total number of inbredbackcross lines, and $z=1.96$ is the 0.95 quantile of the standard normal distribution, with minor exceptions at $c=0,1, \ell-1$, and $\ell$ (Blyth and Still 1983). [7] along with [5] were used to give a $95 \%$ confidence interval for $g$ in the current study.

\section{Results and Discussion}

\section{Year Effects}

Graphs of the line means and $95 \%$ tolerance ellipses about the recurrent parent are presented in Figure 1. Most if not all of the lines fall within the probability ellipse for each trait observed. Also, there are no strong discontinuities in the data from either year for any trait.

If the variance is similar each year, the ellipse is nearly circular, as seen for weight (Figure 1a). Both the range of means and the error variance were slightly higher in 1985 than in 1986 for firmness, resulting in a more elongated ellipse (Figure 1b). The mean square error for soluble solids was much higher in 1985 than 1986, as well as being heterogeneous from line to line (Figure 1c). The line means for soluble solids were somewhat higher in 1985 as well, with both ends of the range about $1.3^{\circ}$ Brix higher. This is probably attributable to a wetter season the second year. Date of first color showed a similar variance each year, but the range of mean dates was much greater in 1985 (Figure 1d). Starting four days later, the last line to show color was 14 days later than the last line to show color in 1986.

\section{Choice of Test}

The use of a tolerance interval to classify inbred-backcross lines into parental and non-parental 
types is inappropriate, since it describes the region of plausible population values but provides no test of significant difference from or equality with the recurrent parent. It could be argued that a test of significant difference from the recurrent parent is appropriate (Rau 1989), but because we are trying to delineate a parental group which does not differ from the parent at any gene, it seems most reasonable to use a confidence interval. If an inbred-backcross line does not differ from the recurrent parent at any gene affecting the trait we would expect it to have the same true mean and variance as the recurrent parent and the sample mean should fall within a $95 \%$ confidence interval about the mean of the recurrent parent. For decisions affected by the classification of all lines it may be desirable to control the overall rate of misclassification.

\section{Comparison of the Tests}

Fewer points will be declared not significantly different from the recurrent parent than would be included in the tolerance ellipse about the recurrent parent. This can be shown by considering one dimension. For the tolerance ellipse, the distance from the center to the edge (half the length of the axis) is the square root of the right-hand side of the equation times the standard deviation. This is approximately $2.45 s_{X}$, using $\chi^{2}$ in the equation as previous authors have. Using our values gives 2.46 $s_{X}\left(2.48 s_{x}\right.$ for color date). This is the distance by which the two means must be separated for the line mean to be outside the ellipse. For a probability rectangle the equivalent distance is $t_{0.0253} s_{x}$ or $2.24 s_{x}$

Hotelling's $T^{2}$ will always give a result that is less than this, $\left(1 / n_{p}+1 / n_{\ell}\right)^{\frac{1}{2}}\left(2.45 s_{x}\right)$. For a twosample $t$ rectangle the distance necessary for significance in one year is $\left(1 / n_{p}+1 / n_{\ell}\right)^{\frac{1}{2}}\left(2.24 s_{x}\right)$. A confidence ellipse would give a much smaller interval than any of these methods, $\left(1 / n_{p}\right)^{\frac{1}{2}}\left(2.45 s_{x}\right)$. For a confidence rectangle the distance is $\left(1 / n_{p}\right)^{\frac{1}{2}}\left(2.24 s_{x}\right)$.

If it was desired to control the overall error rate, a two-sample $t$ rectangle would use the constant 3.04 instead of 2.24 for 55 offspring lines (Appendix). These would achieve an overall error rate of .8 and a comparison-wise error rate of 0.998 .

The rectangle is slightly shorter in length and width than the ellipse, but due to inclusion of the corners the area is almost equal. The choice of which to use would be made on the basis of whether 
the data were paired or unpaired.

\section{Implications to Gene Number Estimates Using Inbred-Backcross Lines}

Since the confidence rectangle delineates a far smaller area than the tolerance ellipse, the number of lines properly classified as parental is drastically reduced. The assertion has been made, both by Wehrhahn and Allard (1965) and by Mulitze and Baker (1985a), that all lines within the tolerance ellipse share the parental genotype. Yet the information presented here shows that the means of many of those lines are actually different from that of the recurrent parent, and that these lines should not be regarded as the same genotype as the recurrent parent. Following Wehrhahn and Allard's (1965) method, ellipses of the same size as the parental tolerance ellipse have been used to delineate putative single gene deviate groups. In many cases lines within these putative groups could be shown to be different from one another. The overall result is that far more genes would be required to account for the observed variability, if it were possible to delineate putative groups. With Mulitze and Baker's (1985a) method, the larger proportion of non-parental types translates into a higher estimate of gene number.

A summary of the results of the six different methods of dividing line means into parental and non-parental types are shown in Tables 1 to 4 for the four traits measured. For the tolerance ellipse and rectangle and confidence ellipse and rectangle, line means falling within the area delineated are classified as parental while those outside are classified as non-parental. For the other two tests, lines not significantly different from and significantly different from the recurrent parent at 0.05 are classified as parental and non-parental respectively. Most if not all of the line means fall within a $95 \%$ tolerance ellipse drawn about the recurrent parent. Very few of the line means fall within a $95 \%$ confidence ellipse drawn about the recurrent parent. For most of the traits, about one third of the lines were declared not significantly different from Calypso by Hotelling's $T^{2}$. There is good agreement between each of these and the equivalent rectangular tests. Using the two-sample $t$ rectangle that controls the overall error rate, about one half of the lines were declared not significantly different from Calypso.

As expected, the estimates of gene number are higher for those methods where more lines are 
classified non-parental. For our case, no genes could be detected for any trait except color date with the tolerance ellipses used by previous authors, but 14 to 30 genes are estimated for the four traits using confidence rectangles, with a wide confidence interval around each estimate.

The numbers calculated should be regarded as a general indication of the numbers of loci involved, because there are many other problems with the method, as discussed by Mulitze and Baker (1985a,b). Using a computer simulation of an inbred-backcross program, they found both upward and downward biases in the estimates of number of loci depending on the heritability of the trait, the level chosen, the number of backcrosses, and sample size. Generally, a low actual number of genes tended to be overestimated and a high actual number underestimated, but the point where an accurate number would be given depended on the combination of the above factors. Based on their results, it is likely that the numbers in the confidence rectangle column are underestimates of the true number of loci involved. It would be interesting to test lines isogenic for a known single gene, for example ovate (o) in tomato, which affects a trait which can be measured quantitatively, to see if the method is able to detect the single gene as predicted by the theory.

After reanalyzing previous results using their method, Mulitze and Baker (1985b) concluded that previous estimates of gene number using the inbred-backcross method were probably too low. Based on a theoretical consideration of the method of classification of lines into parental and non-parental groups and the example of our data it is obvious that the estimates of gene number need to be revised upward again. This number represents only the number of genes by which two homozygous lines differ, which may be a very small portion of the total variability available for a particular trait. Given the uncertainty of the values obtained, the large number of genes apparently involved in all the traits studied, which invalidates one of the key assumptions of the theory, it is likely that the inbredbackcross method has limited practical value in estimation of gene number.

\section{Implications to Other Methods of Estimating Gene Number}

The results of this study also call into question the validity of other methods of estimating gene number. The few studies which have compared the inbred-backcross method with other methods of estimating gene number have generally found good agreement between the methods. The four genes 
proposed by Wehrhahn and Allard (1965) agreed closely with previous results of Allard and Harding (1963) that heading date was controlled by a minimum of four genes based on an analysis of $\mathrm{F}_{6}$ to $\mathrm{F}_{8}$ families. A study carried out by Thurling and Vijendra Das (1979) compared inbred-backcross directly with early generation analysis $\left(\mathrm{F}_{2}\right.$ and backcross); the number of genes detected by each method was similar. Mulitze and Baker (1985c), using the method of moments, estimated that 3-8 genes controlled kernel weight and 2-9 controlled grinding time in spring wheat. Using the genotype assay analysis their results were 2-8 genes and 1-6 genes for the same traits. These ranges are similar to the estimates obtained for the same traits by the inbred-backcross method (Talukdar 1972, Baker 1977). Choo and Reinbergs (1982), using the doubled haploid method, estimated that 6-9 genes control heading date in barley, slightly higher that the four genes found by Wehrhahn and Allard (1965) in wheat. On theoretical grounds, Mulitze and Baker (1985a) concluded that the inbredbackcross method is more accurate than other methods. Because the inbred-backcross method, as it has been used, underestimates gene number, it follows that other methods which have yielded similar results may also underestimate gene number. This needs further theoretical and empirical study.

\section{References}

Allard RW and Harding J, 1963. Early generation analysis and prediction of gain under selection in derivatives of a wheat hybrid. Crop Sci 3:454-456.

Baker RJ, 1977. Inheritance of kernel hardness in spring wheat. Crop Sci 17:960-962.

Baker RJ, 1978. Evaluation of the inbred-backcross method for studying the genetics of continuous variation. Can J Pl Sci 58:7-12.

Blyth CE and Still HA, 1983. Binomial confidence intervals. J Amer Statist Assoc 78: 108-116.

Bourne MC, 1973. Use of the penetrometer for deformation testing of foods. J Food Science 38: 720721.

Chay P and Thurling N, 1989. Identification of genes controlling pod length in spring, Brassica napus L; and their utilization for yield improvement. Plant Breeding 103: 54-62.

Choo TM and Reinbergs E, 1982. Estimation of the number of genes in doubled haploid populations of barley (Hordeum vulgare). Can J Genet Cytol 24:337-341. 
Gibbons JD, Olkin I and Sobel M, 1977. Selecting and ordering populations: a new statistical methodology. New York: Wiley.

Johnson NL and Kotz S, 1972. Distributions in statistics: continuous multivariate distributions, Vol.

4. New York: Wiley.

Johnson RA and Wichern DW, 1988. Applied multivariate statistical analysis, New Jersey: Prentice Hall.

Mulitze DK and Baker RJ, 1985a. Evaluation of biometrical methods for estimating the number of genes. 1. Effect of sample size. Theor Appl Genet 69:553-558.

Mulitze DK and Baker RJ, 1985b. Evaluation of biometrical methods for estimating the number of genes. 2. Effect of type-I and type-II statistical errors. Theor Appl Genet 69:559-566.

Mulitze DK and Baker RJ, 1985c. Genotype assay and method of moments analysis of five quantitative traits in a spring wheat cross. Crop Sci 25:162-167.

Owens KW, Bliss FA, and Peterson CE, 1985. Genetic analysis of fruit length and weight in two cucumber populations using the inbred backcross line method. J Amer Soc Hort Sci 110:431-436.

Rau, G, 1989. An evaluation of the inbred backcross method for estimation of gene number and practical breeding in tomato ( $\mathrm{Ph} \mathrm{D}$ dissertation). Ithaca, New York: Cornell University. Russell WK and Stuber CW, 1983. Inheritance of photosensitivity in maize. Crop Sci 23: 935-939. Snedecor JG and Cochran WG, 1980 Statistical methods, 7th ed. Ames, Iowa: Iowa State University Press.

Sullivan JG and Bliss FA, 1983a. Expression of enhanced seed protein content in inbred-backcross lines of common bean. J Amer Soc Hort Sci 108: 787-791.

Sullivan JG and Bliss FA, 1983b. Genetic control of quantitative variation in phaseolin seed protein of common bean. J Amer Soc Hort Sci 108:782-787.

Talukdar BS, 1972. The genetic basis for the difference in seed weight between Thatcher and Selkirk wheats. Can J Genet Cytol 14:667-673.

Thurling N and Vijendra Das LD, 1979. Genetic control of the pre-anthesis development of spring rape (Brassica napus L). II. Identification of individual genes controlling developmental patterns. 
Austral J Agric Res 30: 261-271.

Wehrhahn C, 1964. The genetic basis for differences in quantitative characters between two varieties of Triticum aestivum L. PhD Dissertation, University of California at Davis.

Wehrhahn C and Allard RW, 1965. The detection and measurement of the effects of individual genes involved in the inheritance of a quantitative character in wheat. Genetics 51:109-119.

Wehrhahn C and GCC Tai, 1988. Gene differences in heading date, height, seed weight and seed yield between two pure line varieties of Triticum aestivum L. Theor Appl Genet 76: 341-351.

Wu KK, Sosulski FW, and Wehrhahn C, 1975. The genetic basis for differences in sedimentation value and protein content between two cultivars of Triticum aestivum. Can J Genet Cytol 17:433-439. 


\section{Figure Legend}

Fig. 1. Distributions of inbred-backcross $B C_{2} S_{4}$ line means in 1986 against mean of the same $B C_{2} S_{3}$ lines in 1985 with tolerance ellipse drawn about the mean of the recurrent parent, Calypso. Traits considered are a) $\log _{10}$ of fruit weight $(\mathrm{g})$, b) fruit firmness $(0.1 \mathrm{~mm}$ deformation), c) fruit soluble solids ( ${ }^{\circ}$ Brix), and d) date of first fruit color (days after July 31). The symbols $\mathbf{a}, \boldsymbol{}$, and + indicate means obtained from Calypso, the donor line, and the inbred-backcross lines, respectively. 
Table 1. Number of inbred-backcross lines classified as parental and non-parental by seven different methods with corresponding estimates of gene number affecting fruit weight.

\begin{tabular}{|c|c|c|c|c|c|c|c|}
\hline & $\begin{array}{l}\text { Tol. } \\
\text { ellip. }\end{array}$ & $\begin{array}{l}\text { Conf. } \\
\text { ellip. }\end{array}$ & $\begin{array}{c}\text { Hotel. } \\
\mathrm{T}^{2}\end{array}$ & $\begin{array}{l}\text { Tol. } \\
\text { rect. }\end{array}$ & $\begin{array}{l}\text { Conf. } \\
\text { rect. }\end{array}$ & $\frac{2 s . t-r}{\text { indiv. }}$ & $\frac{\text { ctangle }}{\text { overall }}$ \\
\hline par. & 55 & 1 & 7 & 55 & 1 & 8 & 20 \\
\hline non. & 0 & 54 & 48 & 0 & 54 & 47 & 35 \\
\hline \multicolumn{8}{|c|}{ Estimates of gene number, with lower and upper limits: } \\
\hline Lower & 0 & 17 & 10 & 0 & 17 & 10 & 5 \\
\hline$\hat{\boldsymbol{g}}$ & $\mathbf{0}$ & 30 & 15 & $\mathbf{0}$ & 30 & 14 & 8 \\
\hline Upper & 1 & 52 & 21 & 1 & 52 & 20 & 11 \\
\hline
\end{tabular}


Table 2. Number of inbred-backcross lines classified as parental and non-parental by seven different methods with corresponding estimates of the number of genes affecting fruit firmness

\begin{tabular}{lccccccc}
\hline & $\begin{array}{l}\text { Tol. } \\
\text { ellip. }\end{array}$ & $\begin{array}{c}\text { Conf. } \\
\text { ellip. }\end{array}$ & $\begin{array}{c}\text { Hotel. } \\
\mathrm{T}^{2}\end{array}$ & $\begin{array}{l}\text { Tol. } \\
\text { rect. }\end{array}$ & $\begin{array}{l}\text { Conf. } \\
\text { rect. }\end{array}$ & \multicolumn{2}{c}{ 2s. t-rectangle } \\
indiv. overall
\end{tabular}

Estimates of gene number, with lower and upper limits:

\begin{tabular}{llrrrrrr} 
Lower & 0 & 10 & 6 & 0 & 10 & 6 & 2 \\
$\hat{\boldsymbol{g}}$ & 0 & 14 & 8 & 0 & 14 & 8 & 4 \\
Upper & 1 & 20 & 12 & 1 & 20 & 12 & 6 \\
\hline
\end{tabular}


Table 3. Number of inbred-backcross lines classified as parental and non-parental by seven different methods with corresponding estimates of the number of genes affecting fruit soluble solids

\begin{tabular}{|c|c|c|c|c|c|c|c|}
\hline & $\begin{array}{l}\text { Tol. } \\
\text { ellip. }\end{array}$ & $\begin{array}{l}\text { Conf. } \\
\text { ellip. }\end{array}$ & $\begin{array}{c}\text { Hotel. } \\
\mathrm{T}^{2}\end{array}$ & $\begin{array}{l}\text { Tol. } \\
\text { rect. }\end{array}$ & $\begin{array}{l}\text { Conf. } \\
\text { rect. }\end{array}$ & $\frac{2 s . t-r}{\text { indiv. }}$ & $\frac{\text { ctangle }}{\text { overall }}$ \\
\hline par. & 54 & 2 & 14 & 54 & 2 & 13 & 20 \\
\hline non. & 1 & 53 & 41 & 1 & 53 & 42 & 35 \\
\hline \multicolumn{8}{|c|}{ Estimates of gene number, with lower and upper limits: } \\
\hline Lower & 0 & 15 & 7 & 0 & 15 & 7 & 5 \\
\hline$\hat{\boldsymbol{g}}$ & 0 & 25 & 10 & 0 & 25 & 11 & 8 \\
\hline Upper & 1 & 38 & 14 & 1 & 38 & 15 & 11 \\
\hline
\end{tabular}


Table 4. Number of inbred-backcross lines classified as parental and non-parental by seven different methods with corresponding estimates of the number of genes affecting date of first fruit color

\begin{tabular}{|c|c|c|c|c|c|c|c|}
\hline & $\begin{array}{l}\text { Tol. } \\
\text { ellip. }\end{array}$ & $\begin{array}{l}\text { Conf. } \\
\text { ellip. }\end{array}$ & $\begin{array}{c}\text { Hotel. } \\
\mathrm{T}^{2}\end{array}$ & $\begin{array}{l}\text { Tol. } \\
\text { rect. }\end{array}$ & $\begin{array}{l}\text { Conf. } \\
\text { rect. }\end{array}$ & $\frac{2 s . t-r}{\text { indiv. }}$ & $\frac{\text { ctangle }}{\text { overall }}$ \\
\hline par. & 41 & 5 & 17 & 42 & 4 & 17 & 26 \\
\hline non. & 14 & 50 & 38 & 13 & 51 & 38 & 29 \\
\hline \multicolumn{8}{|c|}{ Estimates of gene number, with lower and upper limits: } \\
\hline Lower & 1 & 12 & 6 & 1 & 13 & 6 & 4 \\
\hline$\hat{\boldsymbol{g}}$ & 2 & 18 & 9 & 2 & 20 & 9 & 6 \\
\hline Upper & 4 & 25 & 12 & 3 & 28 & 12 & 8 \\
\hline
\end{tabular}




\section{Appendix}

To control the overall probability of misclassifying lines as non-parental we need to control the simultaneous probability of the confidence region for all years and all lines. That is, with $\alpha_{y}$ representing the error rate for each year, we wish to find the value of $d$ such that

$$
P\left\{\left|\frac{\bar{x}_{p}-\bar{x}_{\ell}}{\left(s_{x}^{2}\left(\frac{1}{n_{p}}+\frac{1}{n_{\ell}}\right)\right)^{\frac{1}{2}}}\right|<d \quad \text { for all } \ell\right\}=\alpha_{y} .
$$

This requires either numerical computing or access to tables of the multivariate $t$ distribution, e.g., Gibbons et al. 1977, Table A.5. Tables of the multivariate $t$ need to be accessed with degrees of freedom equal to the degrees of freedom used in estimating error and with correlation

$$
\rho=\frac{1}{\frac{n_{p}}{n_{\ell}}+1} .
$$

In our case, $\rho=\frac{1}{3+1}=.25$ and the tables in Gibbons et al. 1977 only cover $\rho=\frac{1}{2}$, so we computed the probabilities numerically. We solved for the appropriate value of $d$ in [A1] by trial and error. Details are available from Charles McCulloch. 


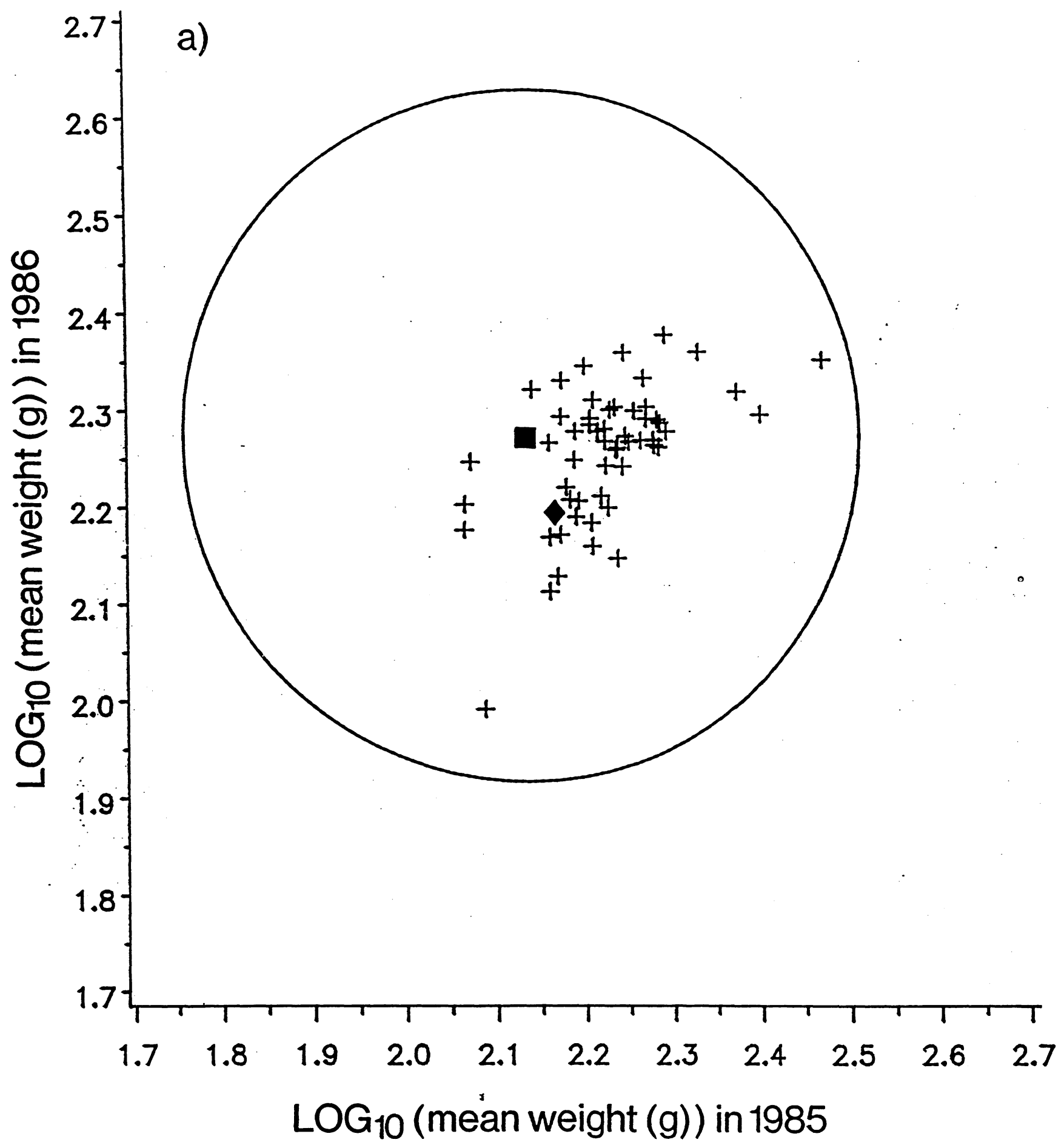




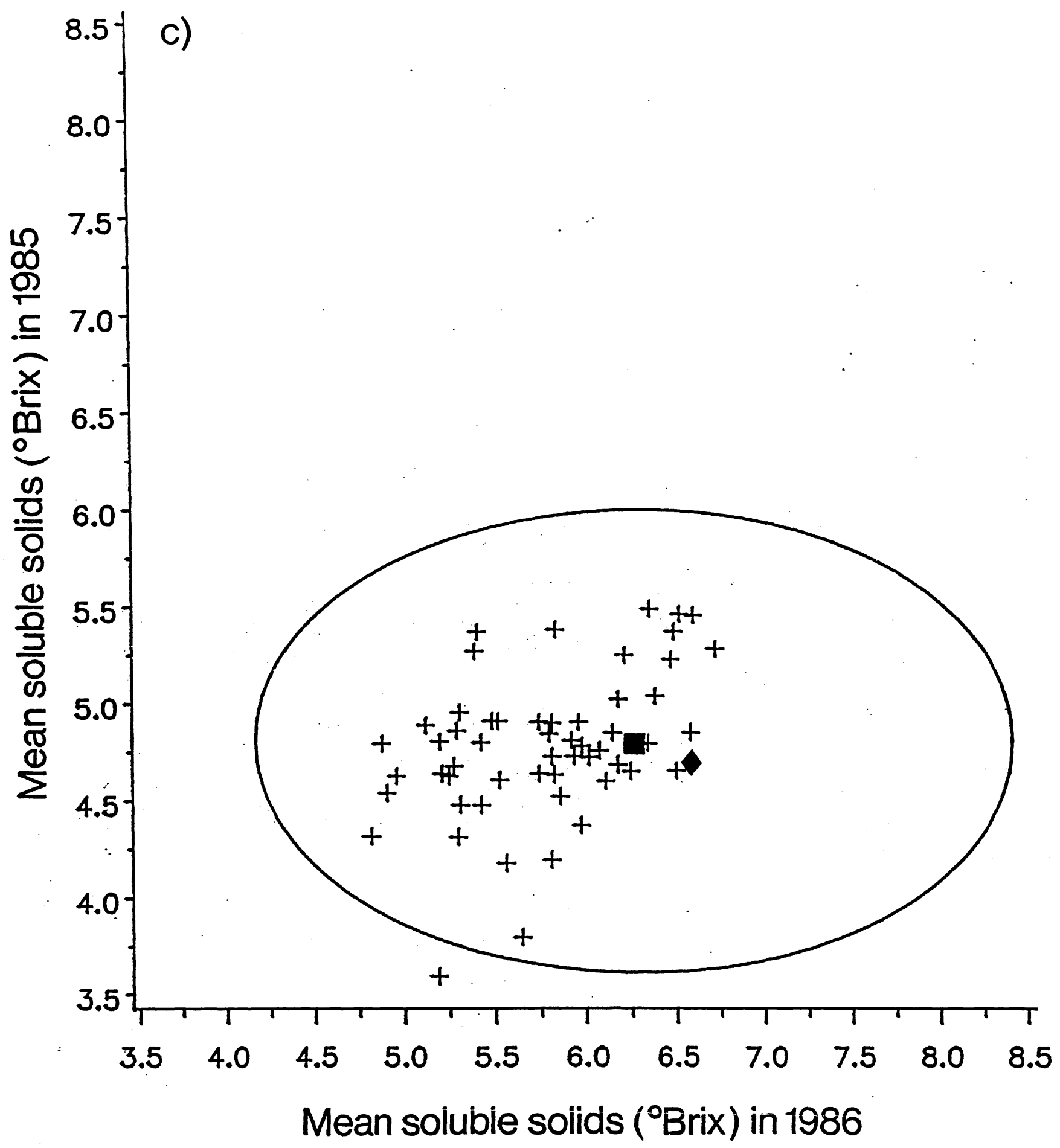




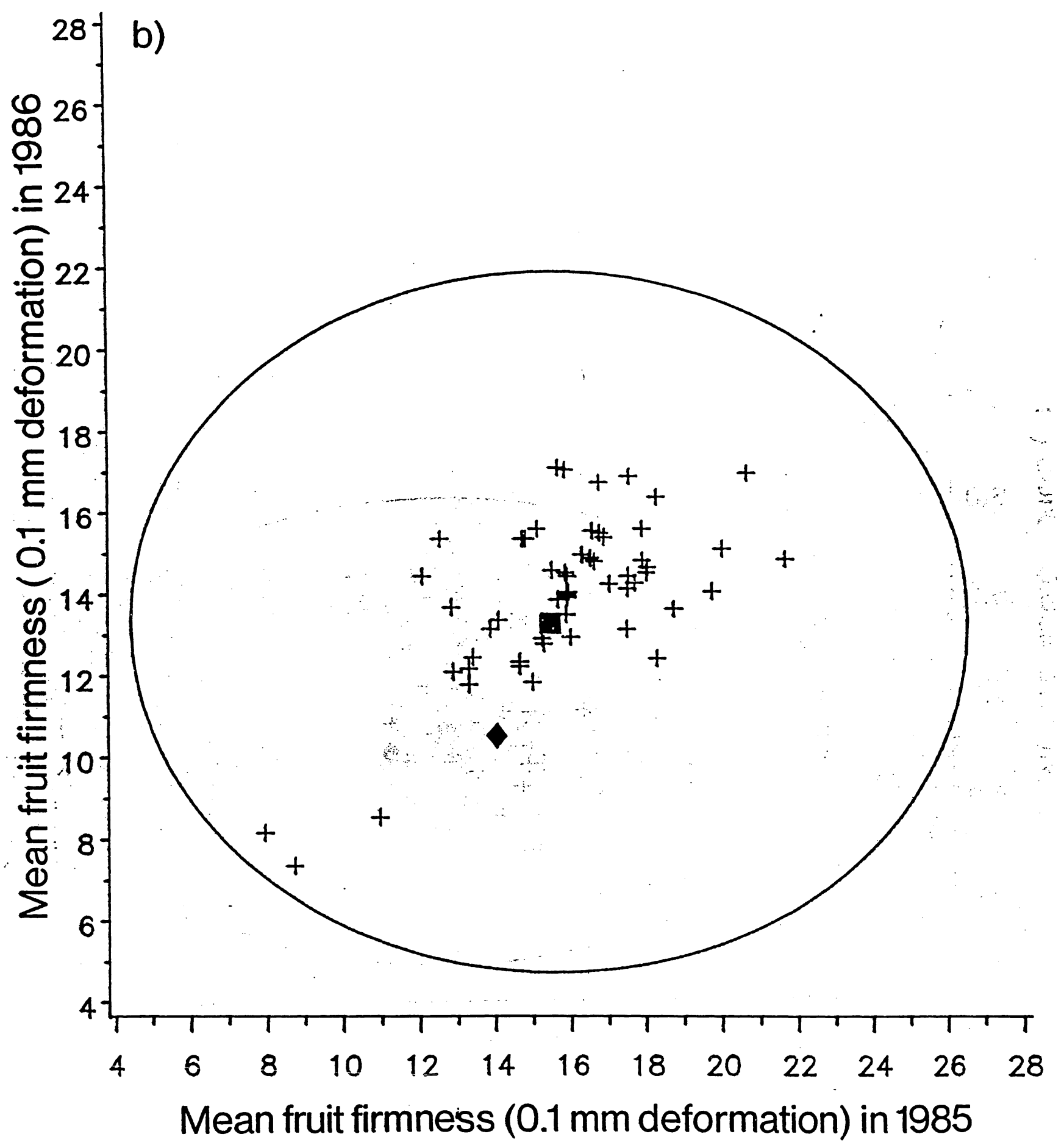




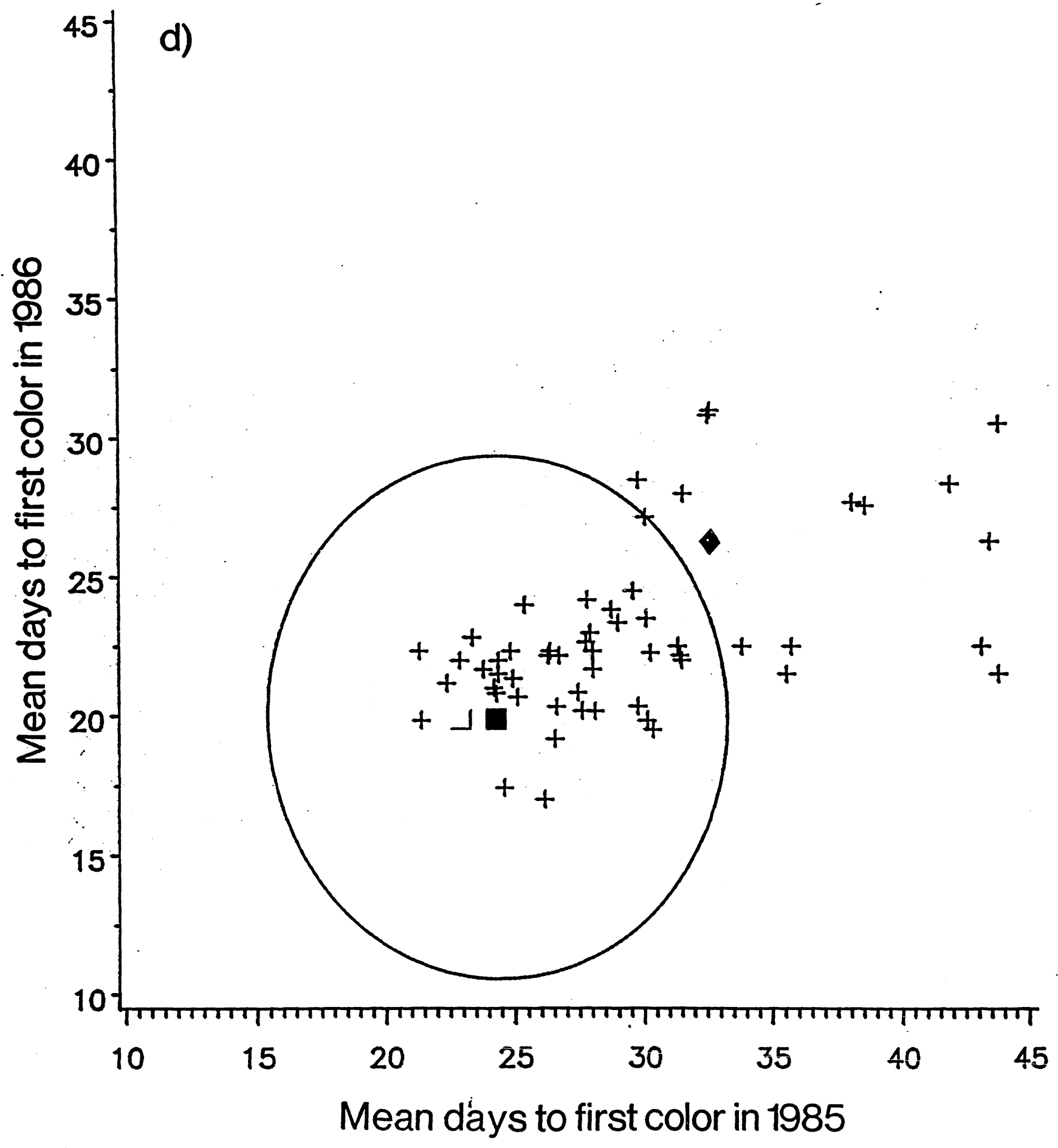

